Z Rheumatol 2015 $74: 288-289$

DOI 10.1007/s00393-014-1478-9

Online publiziert: 10. Mai 2015

c) Springer-Verlag Berlin Heidelberg 2015
K. de Groot ${ }^{1}$ - S.M. Weiner ${ }^{2}$

${ }^{1}$ Sana Klinikum Offenbach, KfH-Nierenzentrum, Offenbach

${ }^{2}$ Krankenhaus der Barmherzigen Brüder, KfH-Nierenzentrum, Trier

\title{
Rheuma und Niere
}

Die Fachdisziplinen Rheumatologie und Nephrologie bieten zahlreiche Überschneidungen und Interaktionen. Dies betrifft insbesondere rheumatische Systemkrankheiten, die häufig mit einer Nierenbeteiligung einhergehen.

Die glomeruläre Beteiligung bei rheumatischen Erkrankungen, wie dem systemischen Lupus erythematodes und der ANCA-assoziierten Vaskulitis, ist gut klassifiziert und über Urindiagnostik sowie Nierenbiopsie einfach zu differenzieren. Hingegen bestehen bei interstitiellen Nephritiden größere Probleme zum einen in der Diagnostik, da Nierenfunktion und Urinsediment nicht immer wegweisend sind, und zum anderen in der Abgrenzung zwischen den verschiedenen Entitäten, einem Thema, dem sich der Beitrag von Korsten et al. widmet.

Ein nicht unerheblicher Anteil der Patienten mit Rheumaerkrankungen weist eine eingeschränkte Nierenfunktion auf. Diese ist oft in lange bestehenden Komorbiditäten begründet (arterielle Hypertonie, Diabetes mellitus, Einnahme von nichtsteroidalen Antirheumatika, Kontrastmittelexposition) und muss nicht kausal im Zusammenhang mit der rheumatischen Erkrankung stehen.

Zur Abschätzung der Nierenfunktion wird meist das Serumkreatinin herangezogen, allerdings sollte stets die geschätzte glomeruläre Filtrationsrate (eGFR) mittels MDRD- oder EPI-Formel errechnet werden. Gerade bei älteren Patienten mit reduzierter Muskelmasse kann das Serumkreatinin noch lange im Normbereich liegen, obwohl bereits eine deutlich eingeschränkte Nierenfunktion vorliegt. Bei muskelschwachen Patienten und Kindern ist deshalb zusätzlich die Bestimmung von Cystatin C sinnvoll, wodurch die Nierenfunktion genauer abgeschätzt werden kann.

Die Einteilung der Niereninsuffizienz wurde 2013 von der KDIGO (Kidney Disease: Improving Global Outcomes) revidiert, hierbei werden 5 GFR- und 3 Albuminuriestadien unterschieden (• Tab. 1). Neu gegenüber der früheren Einteilung ist, dass das Stadium 3 (GFR 30-60 ml/ $\mathrm{min}$ ) der Niereninsuffizienz in G3a (45$60 \mathrm{ml} / \mathrm{min})$ und $\mathrm{G} 3 \mathrm{~b}(30-45 \mathrm{ml} / \mathrm{min})$ unterteilt wird und dass die Proteinurie mit einfließt. Dies liegt darin begründet, dass die Gesamtmortalität und insbesondere die kardiovaskuläre Mortalität bei einer GFR $<45 \mathrm{ml} / \mathrm{min}$ deutlich ansteigt [1] und auch die Albuminurie eine prognostische Bedeutung hat.

Bei Patienten mit rheumatischen Erkrankungen werden die Auswahl und Dosierung von Basismedikamenten, Immunsuppressiva, Analgetika sowie Osteoporosemedikation durch eine Niereninsuffizienz erheblich beeinflusst. Einen Überblick über die Möglichkeiten und Grenzen der Rheumatherapie bei Niereninsuffizienz geben Weiner u. Bergner.
Die Nierenfunktion hat auch einen nicht unerheblichen Einfluss auf die bildgebende und laborchemische Diagnostik. Beispiele sind die Verwendung von Röntgenkontrastmitteln in der CT-Diagnostik (Risiko des kontrastmittelinduzierten Nierenversagens) und von Gadolinium im MRT bei schwer eingeschränkter Nierenfunktion (nephrogene systemische Fibrose) sowie die fehlende Vorhersagekraft der DEXA-Osteodensitometrie an der Wirbelsäule bei einer GFR $<30 \mathrm{ml} / \mathrm{min}$ hinsichtlich des Frakturrisikos. Andererseits kommt der Knochenbiopsie zur Therapieplanung der Osteopathien eine zentrale Rolle zu. Parathormon, Knochenphosphatase, Vitamin-DStatus, Serumcalcium- und -phosphat, venöse Blutgasanalyse, Eisenstatus und Retikulozyten sind wichtige Parameter hinsichtlich der Therapieplanung bei chronischer Niereninsuffizienz.

Die Nierenfunktion ist nicht nur für die Therapie, sondern auch für die Morbidität und Mortalität der Patienten, die an rheumatischen Erkrankungen leiden, von erheblicher Bedeutung. Das Risiko

Tab. 1 Revidierte Klassifikation der chronischen Niereninsuffizienz basierend auf der glomerulären Filtrationsrate (GFR) und der Albuminurie. (Nach [1])

\begin{tabular}{lll}
\hline GFR-Stadien & GFR $\left(\mathbf{m l} / \mathbf{m i n} / \mathbf{1}, \mathbf{7 3} \mathbf{~ m}^{2}\right)$ \\
\hline G1 & $>90$ & Normal oder hoch \\
\hline G2 & $60-89$ & Leicht vermindert \\
\hline G3a & $45-59$ & Leicht bis mäßig vermindert \\
\hline G3b & $30-44$ & Mäßig bis schwergradig vermindert \\
\hline G4 & $15-29$ & Schwergradig vermindert \\
\hline G5 & $<15$ & Nierenversagen (D hinzufügen bei Dialysebehandlung) \\
\hline Albuminuriestadien & Albuminausscheidung (mg/Tag oder $\mathbf{m g} / \mathbf{g}$ Kreatinin) \\
\hline A1 & $<30$ & Normal bis leicht erhöht \\
\hline A2 & $30-300$ & Mäßig erhöht \\
\hline A3 & $>300$ & $\begin{array}{l}\text { Schwergradig erhöht (unterteilt in nephrotisch und } \\
\text { nichtnephrotisch) }\end{array}$ \\
\hline
\end{tabular}


Tab. 2 Zielblutdruck bei Niereninsuffi-

zienz in Abhängigkeit von der Albuminurie. (Nach [1])

\begin{tabular}{|lll}
\hline Albuminurie & Zielblutdruck & $\begin{array}{l}\text { Evidenz- } \\
\text { level }\end{array}$ \\
\hline$<30 \mathrm{mg} / 24 \mathrm{~h}$ & $\leq 140 / 90 \mathrm{mmHg}$ & $1 \mathrm{~B}$ \\
\hline$\geq 30 \mathrm{mg} / 24 \mathrm{~h}$ & $\leq 130 / 80 \mathrm{mmHg}$ & $2 \mathrm{D}$ \\
\hline$\geq 300 \mathrm{mg} /$ & $\leq 130 / 80 \mathrm{mmHg}$ & $1 \mathrm{~B}$ \\
$24 \mathrm{~h}$ & $\begin{array}{l}\text { Verwendung } \\
\text { von ACE- oder }\end{array}$ \\
& $\mathrm{AT}_{1}$-Blockern \\
\end{tabular}

$\mathrm{zu}$ versterben ist bei Niereninsuffizienz erhöht: eGFR $60 \mathrm{ml} / \mathrm{min} / 1,73 \mathrm{~m}^{2}$ Hazard Ratio (HR) 1,18, eGFR $45 \mathrm{ml} / \mathrm{min} / 1,73 \mathrm{~m}^{2}$ HR 1,57, eGFR $15 \mathrm{ml} / \mathrm{min} / 1,73 \mathrm{~m}^{2} \mathrm{HR} 3,14$ [2]. Auch der Albumin/Kreatinin-Quotient ist ohne Schwellenwert linear mit der Mortalität assoziiert. Ursächlich für die gesteigerte Mortalität bei Niereninsuffizienz sind $u$. a. die akzelerierte Arteriosklerose aufgrund der Störung im Calcium-Phosphat-Haushalt sowie die im Rahmen der Niereninsuffizienz auftretende Mikroinflammation, Anämie und aktive vaskuläre Kalzifikation. Bei fortgeschrittener Niereninsuffizienz kommt es zudem zur zellulären Immundefizienz, die mit einer ungenügenden Impfantwort und einem erhöhten Risiko für bakterielle Infektionen einhergeht. Dies muss auch beim Einsatz von Immunsuppressiva und Kortikosteroiden berücksichtigt werden. Der Beitrag von Zänker et al. gibt einen Überblick über das Outcome von Patienten mit entzündlich-rheumatischen Erkrankungen mit Niereninsuffizienz und die daraus resultierenden Konsequenzen für die Therapie.

Bei Patienten mit Niereninsuffizienz sind präventive Maßnahmen zur Progressionsverzögerung erforderlich. Neben einer Blutdrucksenkung (• Tab. 2) werden weitere Maßnahmen empfohlen, insbesondere Lebensstilinterventionen wie Reduktion der Salzaufnahme $<6$ g/Tag, Anstreben eines Body-Mass-Indexes von 20-25, Beenden eines Nikotinkonsums und optimale altersadaptierte Diabeteseinstellung mit einem Ziel-HbA $\mathrm{H}_{1 \mathrm{c}}$ von 7\% [1]. Bei proteinurischen Patienten sollen primär ACE-Hemmer oder $\mathrm{AT}_{1}$-Blocker zum Einsatz kommen. Die Kombination von $\mathrm{ACE}-H e m m e r n$ mit $\mathrm{AT}_{1}$-Blockern oder von ACE-Hemmern bzw. $\mathrm{AT}_{1}$-Blockern mit Aldosteronantagonisten bleibt
Patienten mit refraktärer großer Proteinurie vorbehalten.

Auf neue Erkenntnisse zur Bedeutung der Hyperurikämie, einem häufigen Begleitphänomen der Niereninsuffizienz, wird in der Übersicht von Sellin et al. gesondert eingegangen. Epidemiologische Studien belegen, dass sowohl die Gicht als auch in schwächerem Ausmaß die asymptomatische Hyperurikämie mit einer Verschlechterung der Nierenfunktion, vermehrten vaskulären Komplikationen sowie einem erhöhten Risiko für Mortalität assoziiert sind.

Der fachliche Austausch zwischen Rheumatologie und Nephrologie ist in Kliniken, in denen beide Fächer unter einem Dach vereint sind, unproblematisch; ansonsten ist eine intensive interdisziplinäre Zusammenarbeit zum Wohle der betroffenen Patienten sicherlich sinnvoll.

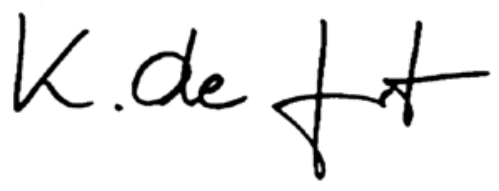

K. de Groot
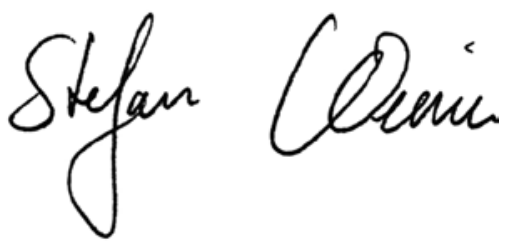

S.M. Weiner

\section{Korrespondenzadresse}

Prof. Dr. K. de Groot

Sana Klinikum Offenbach, KfH-Nierenzentrum Starkenburgring 66, 63069 Offenbach

kirsten@de-groot.de

Prof. Dr. S. M. Weiner

Krankenhaus der Barmherzigen Brüder, KfH-Nierenzentrum

Nordallee 1,54292 Trier

s.weiner@bk-trier.de

Interessenkonflikt. K. de Groot erhielt Vortragshonorare von Abbot, Amgen, BMS, Berlin Chemie, Jansen-Cliag, Pfizer, Roche, UCB und übt Beratungstätigkeit für die Fa. Amgen und Roche aus; S.M. Weiner erhielt im Rahmen von Vorträgen Honorare der Firmen Abbott, BMS, MSD, Pfizer und Roche.

\section{Literatur}

1. Stevens PE, Levin A; Kidney Disease: Improving Global Outcomes Chronic Kidney Disease Guideline Development Work Group Members (2013) Evaluation and management of chronic kidney disease: synopsis of the kidney disease: improving global outcomes 2012 clinical practice guideline. Ann Intern Med 158(11):825-830

2. Chronic Kidney Disease Prognosis Consortium, Matsushita K, van der Velde M, Astor BC et al. (2010) Association of estimated glomerular filtration rate and albuminuria with all-cause and cardiovascular mortality in general population cohorts: a collaborative meta-analysis. Lancet 375(9731):2073-2081 